\title{
Design of Hybrid Neural Fuzzy Controller for Human Robotic Leg System
}

\author{
Ekhlas H. Karam* Ayam M. Abbass** Noor S. Abdul-Jaleel*** \\ *, **Department of Computer Engineering/ University of Al-Mustansyria \\ ***Department of Electrical Engineering/ University of Al-Mustansyria \\ *Email: ekhlashameed@yahoo.co.uk \\ **Email: ayammohsen@yahoo.com \\ ***Email: mail_ns1@yhoo.com
}

(Received 10 January 2017; accepted 7 August 2017)

https://doi.org/10.22153/kej.2018.08.007

\begin{abstract}
In this paper, the human robotic leg which can be represented mathematically by single input-single output (SISO) nonlinear differential model with one degree of freedom, is analyzed and then a simple hybrid neural fuzzy controller is designed to improve the performance of this human robotic leg model. This controller consists from SISO fuzzy proportional derivative (FPD) controller with nine rules summing with single node neural integral derivative (NID) controller with nonlinear function. The Matlab simulation results for nonlinear robotic leg model with the suggested controller showed that the efficiency of this controller when compared with the results of the leg model that is controlled by PI+2D, PD+NID, and FPD-ID controllers.
\end{abstract}

Keywords: Fuzzy proportional derivative controller, Matlab simulation results, Nonlinear differential leg model, PID controller, single node neural controller.

\section{Introduction}

The classical robots are commonly used for industrial automation and use in applications that are remote from human life and activities. However, recently the usage of robots has been changed from industrial applications to helpful for human robot system. With increasing aging societies, robots that used to support human in his activities in daily environments such as in offices, homes, school and hospitals are expected. Particularly, because of anthropomorphism, helpful design for humanity, application of positioning and movement, and so on, powerfully expected to manufacture of humanoid robots [1].

Present control techniques to locomotion of robotic legged depends on centralized planning and path follow or corresponding motion pattern. Central control is not available to the robotic help devices that make integration with humans, and correspond to predefined patterns strongly limits from user ability. By difference, biological systems show big legged ability even when the system of the central nervous is separated from spinal cord that belong to these legged, pointing that feedback controls of neuromuscular is harnessed for encoding stability, compatibility, and maneuverability into robotic legged systems [2].

Currently, control systems design is done by a big number of requirements caused by augmentation of competition, requirements of environment, energy and the costs of components and the request for robustness, system of error tolerance. These considerations introduce extra 
needs for effective process modeling techniques. Several systems are not agreeable to traditional modeling approaches, caused when the precision is not found, about the system having formal knowledge, caused from strong behavior for nonlinearities, big degree of suspicion, or variance characteristic of the time [3].

Neural networks and fuzzy logic systems are recently used for different control problems with acceptable results. Both the neural networks and fuzzy logic systems are general approximations caused several adaptive control strategies for nonlinear systems that used fuzzy logic systems, or neural networks have been introduced to get more control performance [4].

As two important strategies of artificial intelligence, fuzzy logic systems and Artificial Neural Networks (ANNs) have several applications in different fields such as availability of product, control systems, diagnostic, observation, etc. They developed and became better throughout the years for adaptation of the rising requirements and the develop of technology. While these two controllers have been frequently used together, explain that the concept of a fusion began to take shape [5].

The neuro-fuzzy system is more efficient and more effective than either neural network or fuzzy logic system which has been widely applied in control systems, pattern recognition, medicine, expert system, and etc. The advantage of this controller is the dealing with it more quickly than other traditional controllers [6].

Different types of neuro-fuzzy have been shown in the literature. These types can be determined depend on the structure of the neurofuzzy, the fuzzy model used, and the learning algorithm taken. On the first hand, corresponding to the neuro-fuzzy structure and learning algorithm, the most usually used and successful technique is the feed-forward and recurrent structure model using the BP learning algorithm. On the other hand, according to the fuzzy model taken, two types of fuzzy models are merged with a neural network to form a neuro-fuzzy. These two models are familiar as Takagi and Sugeno model and the Mamdani-model. However, Mamdani-model based NNF represent more obvious neuro-fuzzy systems compared with TSmodel based NNF [7].

A simple hybrid neural fuzzy controller is designed in this paper for human robotic leg model, this controller consist from single node neural integral derivative (NID) controller and single input single output fuzzy proportional derivative (FPD) controller with nine rules. The details for this design will be explained in the sections of this paper.

\section{Human Robotic Leg Mathematical Model}

The human robotic leg can by modeled depend on the relation between the input torque that generated by the muscle of the leg and the output of the angular rotation around the hip joint [8]. A simplified human robotic leg cylindrical model is shown in Fig. 1.

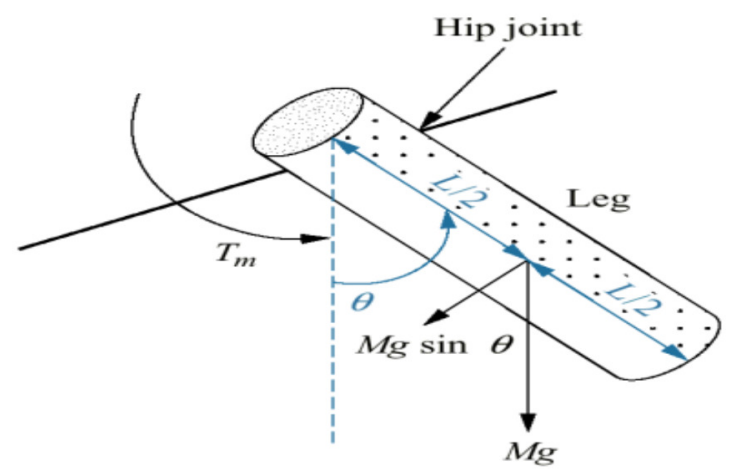

Fig. 1. Human a robotic leg cylindrical model [9].

The parameters of Fig. 1 are defined by Table 1 .

Table 1,

The parameters of human robotic leg model.

\begin{tabular}{lll}
\hline Symbol & Description & values \\
\hline $\mathrm{T}_{\mathrm{m}}$ & $\begin{array}{l}\text { torque supplied by DC } \\
\text { motor }\end{array}$ \\
$\mathrm{D}$ & $\begin{array}{l}\text { viscous damping } \\
\end{array}$ & $\begin{array}{l}0.1 \mathrm{Nms} / \mathrm{ra} \\
\mathrm{d}\end{array}$ \\
$\mathrm{J}$ & inertia around the hip joint & $\begin{array}{l}0.4 \\
\mathrm{kgm}^{2} / \mathrm{s}^{2}\end{array}$ \\
$\mathrm{M}$ & mass of the leg & $1 \mathrm{~kg}$ \\
$\mathrm{~g}$ & $\begin{array}{l}\text { acceleration due to gravity } \\
\text { creates a nonlinear torque } \\
\text { the length of the leg and the }\end{array}$ & 9.81 \\
$\mathrm{~L}$ & $0.5 \mathrm{~m}$ \\
& $\begin{array}{l}\text { weight can be determine as } \\
\end{array}$ \\
& L/2 \\
\hline
\end{tabular}

The nonlinear equation of the dynamic model for the robotic human leg can be written as follows $[9,10]$ :

$J \frac{d^{2} \theta}{d t^{2}}+D \frac{d \theta}{d t}+M g \frac{L}{2} \sin \theta=T_{m}(t)$

Where: $\operatorname{Mg} \frac{\mathrm{L}}{2} \sin \theta$ is the component of weight, $\mathrm{D} \frac{\mathrm{d} \theta}{\mathrm{dt}}$ is the damping torque and $\left(\frac{d^{2} \theta}{d t^{2}}\right)$ is the inertia torque. The state space for Eq. (1) is: 
$\left[\begin{array}{l}\dot{x_{1}} \\ \dot{x_{2}}\end{array}\right]=\left[\begin{array}{cc}0 & 1 \\ -\left(\frac{1}{x 1} \frac{M g L}{2 J} \sin x_{1}\right) & -\left(\frac{D}{J}\right)\end{array}\right]\left[\begin{array}{l}x_{1} \\ x_{2}\end{array}\right]+\left[\begin{array}{l}0 \\ \frac{1}{J}\end{array}\right] T_{m}$ $y=\left[\begin{array}{ll}1 & 0\end{array}\right]\left[\begin{array}{l}x_{1} \\ x_{2}\end{array}\right]$

Where: $\mathrm{x}_{1}, \mathrm{x}_{2}$ is robotic leg angular position and velocity respectively. Eq. (1) can be linearized for small angle approximation, the $\theta$ variation is small; therefore $\sin \theta$ can assume equal $\theta$ (i. e. $\sin \theta=\theta$ ) so Eq. (1) becomes:

$J \frac{d^{2} \theta}{d t^{2}}+D \frac{d \theta}{d t}+M g \frac{L \theta}{2}=T_{m}(t)$

The linear transfer function of Eq. (3) is given by:

$\frac{\theta(s)}{T(s)}=\frac{\frac{1}{J}}{s^{2}+\frac{D}{J} s+\frac{M g L}{2 J}}$

With parameter given by Table 1, Eq. (4) becomes:

$\frac{\theta(s)}{T(s)}=\frac{2.5}{s^{2}+0.025 s+6.131}$

This transfer equation has two complex poles $(-0.0125+2.4761 \mathrm{j},-0.0125-2.4761 \mathrm{j})$ with damping ratio $\zeta=0.0050$, the state space equations are:

$\left[\begin{array}{l}\dot{x_{1}} \\ \dot{x_{2}}\end{array}\right]=\left[\begin{array}{cc}0 & 1 \\ -6.131 & -0.025\end{array}\right]\left[\begin{array}{l}x_{1} \\ x_{2}\end{array}\right]+\left[\begin{array}{c}0 \\ 2.5\end{array}\right] T_{m}$
$y=\left[\begin{array}{ll}1 & 0\end{array}\right]\left[\begin{array}{l}x_{1} \\ x_{2}\end{array}\right]$

With unity step input, the time response of the leg linear and nonlinear model is shown in Fig. 2.

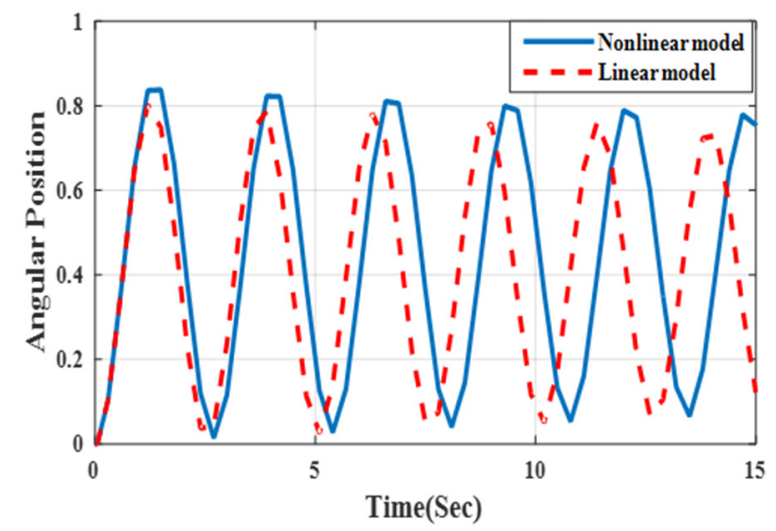

Fig. 2. Time response for linear and nonlinear model.

This figure show that the leg model response is high oscillation under damped. In order to reduce the oscillation, a suitable velocity feedback gain $\left(\mathrm{K}_{\mathrm{v}}=3.74\right)$ is adding to plant leg transfer function and hence this function becomes:

$\frac{\theta(s)}{T(s)}=\frac{2.5}{s^{2}+\left(0.025+2.5 K_{v}\right) s+6.131}=\frac{2.5}{s^{2}+8.7 s+6.131} .$.

The damping ratio for linear model becomes $\zeta=1.76$, so the response of unity step input for this equation and for the nonlinear model become over damped as shown in Fig. 3.

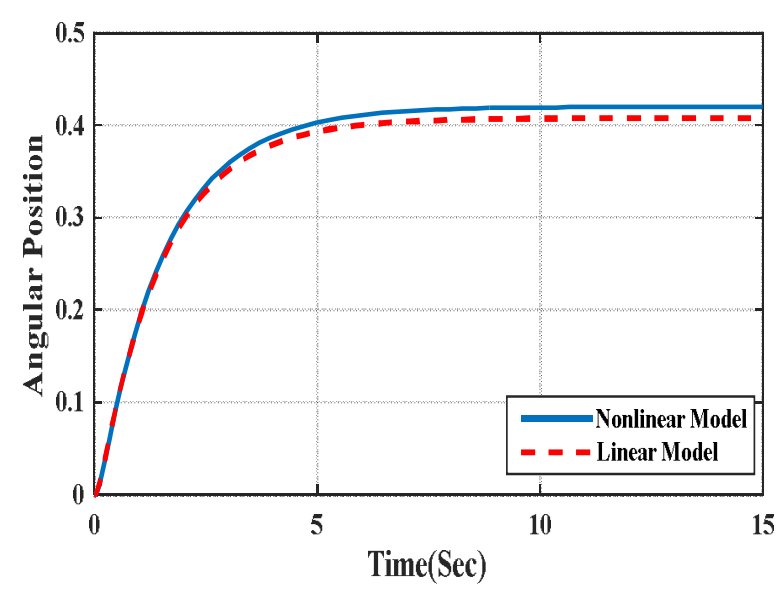

Fig. 3. Time response for linear and nonlinear model with velocity feedback gain $k_{v}=3.74$

\section{Hybrid Neural Fuzzy Controller Design}

The block diagram for nonlinear robotic leg model with the suggested hybrid like neural fuzzy controller is shown in Fig. 4.

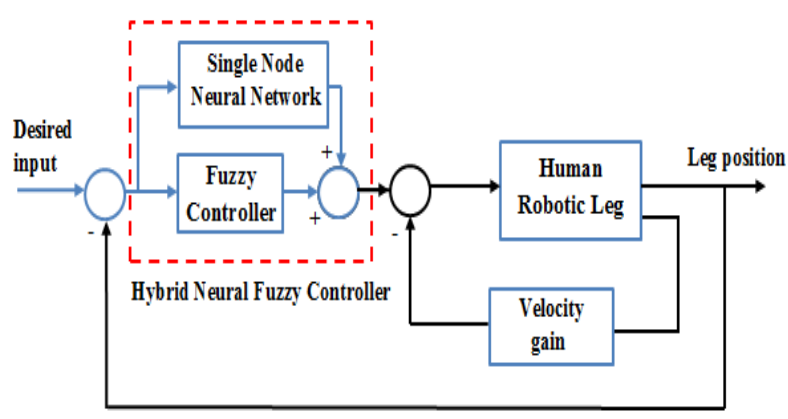

Fig. 4. The block diagram for hybrid neural fuzzy controller for human robotic leg.

The suggested hybrid controller consists from SISO neural integral derivative (NID) and fuzzy proportional derivative (FPD) controller. The first step for design this controller is determine the proportional integral derivative (PID) controller for the robotic leg based on the linear model of the robotic leg.

Generally, the equation of the ideal PID controller with ideal form is given as:

$U(s)=\left(K_{p}+\frac{K_{i}}{s}+K_{d}(s)\right) E(s)$

Where: $\mathrm{E}(\mathrm{s})$ is the error signal between the desired input and the leg output position, while $\mathrm{K}_{\mathrm{p}}, \mathrm{K}_{\mathrm{i}}$, and $\mathrm{K}_{\mathrm{d}}$ are proportional, integral and 
derivative gain in respectively, the values of these parameters are determine by Launching PID tuner method that provide by matlab simulink with some manual modification. Eq. (8) can be written as:

$U(s)=K_{p}\left(1+\frac{1}{T_{i} s}+T_{d} s\right) E(s)$

Where: $T_{i}$ is integral or reset time, and $T_{d}$ is derivative or rate time.

In this paper, instead of ideal PID controller we use PI+2D and hence Eq. (9) becomes:

$U(s)=K_{p}\left(1+\frac{1}{T_{i} s}+T_{d} s+T_{d} s\right) E(s)$

Or

$U(s)=K_{p}\left(\left(1+T_{d} s\right)+\left(\frac{1}{T_{i} s}+T_{d} s\right)\right) E(s)$

Eq. (11) can be written as:

$$
\begin{aligned}
U(s) & =K_{p}\left(1+T_{d} s\right) E(s)+K_{p}\left(\frac{1}{T_{i} s}+T_{d} s\right) E(s) \\
& =U_{1}(s)+U_{2}(s)
\end{aligned}
$$

The steps for designing the suggested controller will be demonstrate in the following sub sections.

\subsection{Fuzzy Proportional Derivative Controller Design}

The first part of Eq. (12) is ideal PD controller as given by:

$U_{1}(s)=K_{p}\left(1+T_{d} s\right) E(s)$

This equation is considered as input to the SISO fuzzy controller as shown in Fig. 5 after remove $K_{\mathrm{p}}$ as given by:

$u_{i}(t)=\operatorname{sat}\left(\left(1+T_{d} \frac{d}{d t}\right) e(t)\right)$

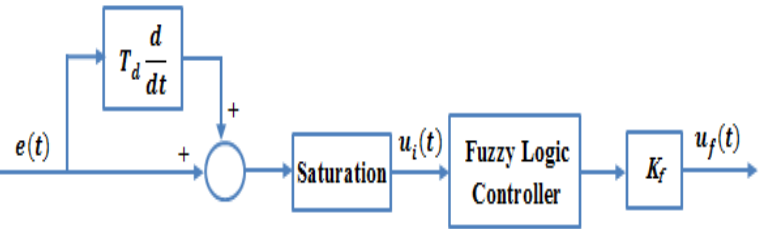

Fig. 5. Fuzzy PD logic controller.

The saturation function is used to limit any input signal to be between ( $\mathrm{N}$ to $-\mathrm{N}$ ), $\mathrm{K}_{f}=\mathrm{K}_{p} \times$ $K_{s}$ where $\mathrm{K}_{\mathrm{s}}$ is selected gain such that the value of $\mathrm{K}_{\mathrm{f}}$ should be equal to magnitude of the input signal, for example if the input is step with magnitude 10 , then $\mathrm{K}_{\mathrm{f}}$ should be equal to10.

Usually, fuzzy logic theory deals with uncertain or imprecise situation. A variable in fuzzy logic has sets of values which are characterized by linguistic expressions, such NEGATIVE, ZERO, POSITIVE, etc. These linguistic expressions are represented numerically by fuzzy set and it is characterized by membership function that varies from [0 to 1], which given element can be a member of any fuzzy set [11].

In general, the fuzzy controller design consists from three parts:

\subsubsection{Fuzzification}

The input (crisp) data is fuzzified according to a selected membership functions.

The input and output membership are shown in Fig. 6.

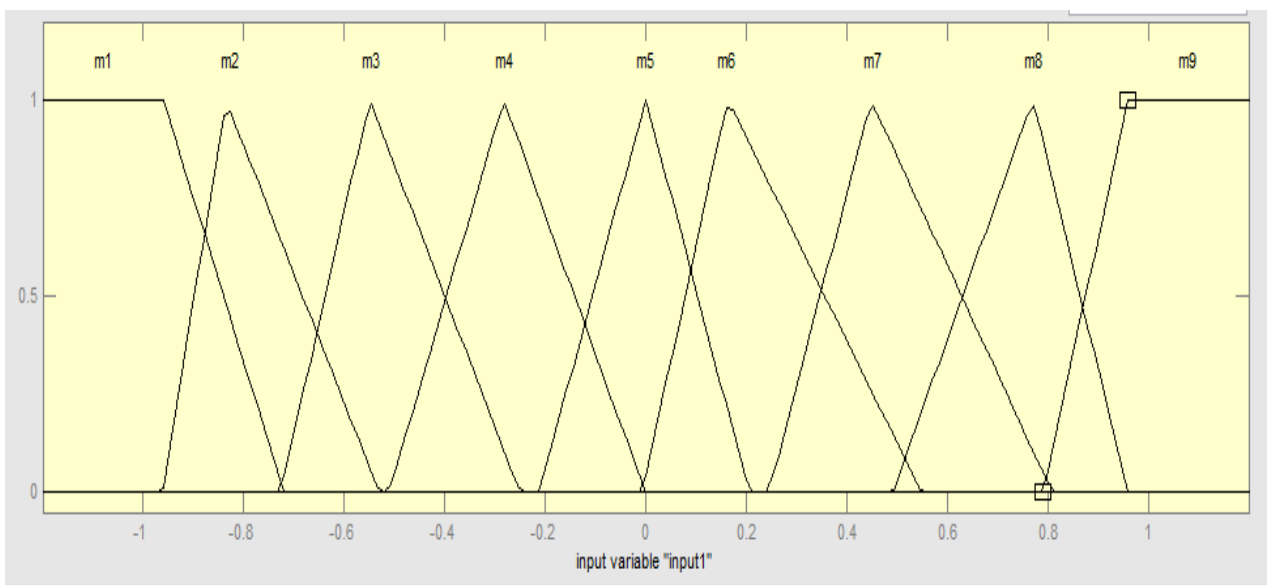




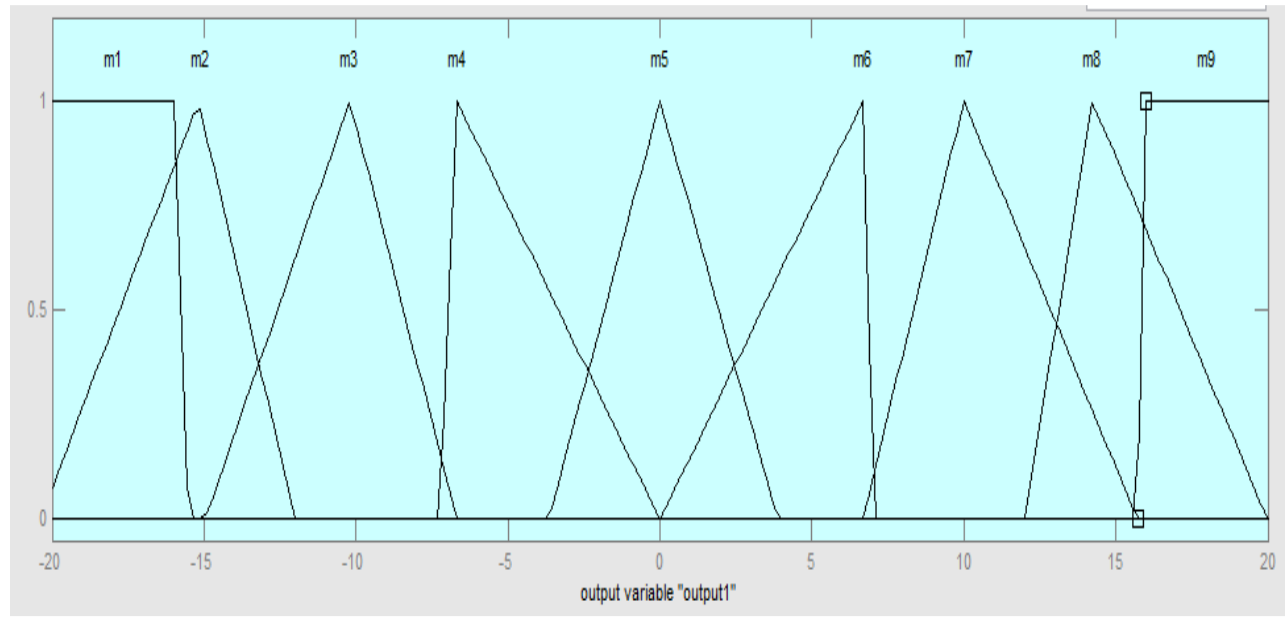

(b)

Fig. 6. Membership function for, (a) input variable, (b) output variable.

\subsubsection{Rule Base}

The knowledge base of the fuzzy logic consists of a set of fuzzy IF (the satisfied conditions set) THEN (the inferred consequence set) rules which themselves consist of linguistic set, associated variables with inputs, outputs and fuzzy operators like OR and AND [12].

The quantitative if-then rules of our fuzzy controller are described as:

R1: if $u_{i}(t)$ is $m 1$ then $u_{f}(t)$ is $m 1$

R2: if $u_{i}(t)$ is $m 2$ then $u_{f}(t)$ is $m 2$

R3: if $u_{i}(t)$ is $m 3$ then $u_{f}(t)$ is $m 3$

R4: if $u_{i}(t)$ is $m 4$ then $u_{f}(t)$ is $m 4$

R5: if $u_{i}(t)$ is $m 5$ then $u_{f}(t)$ is $m 5$

R6: if $u_{i}(t)$ is $m 6$ then $u_{f}(t)$ is $m 6$

R7: if $u_{i}(t)$ is $m 7$ then $u_{f}(t)$ is $m 7$

R8: if $u_{i}(t)$ is $m 8$ then $u_{f}(t)$ is $m 8$

R9: if $u_{i}(t)$ is $m 9$ then $u_{f}(t)$ is $m 9$

Where: $\mathrm{m}$ is linguistic terms of antecedent fuzzy set, they mean $\mathrm{m} 1=\mathrm{mf} 1, \ldots, \mathrm{m} 9=\mathrm{mf} 9$.

The universe of discourse from -1.2 to 1.2 and from -20 to 20 for the input and the output membership function in respectively as shown in Fig. 6.

\subsubsection{Defuzzification}

In this step, the Center of Mass (COM) formula is used to defuzzify the law of the fuzzy controller.

\subsection{Neural Integral Derivative Controller Design}

A neural network is an interconnected network of nodes, working manner similar neurons in the human brains. Each neurons contains a link collector that collects weighted with the shift income to form a numerical output of neuron, which is considered as lacking in movement with neural network $\mathrm{f}_{\mathrm{nn}}(\mathrm{x})$, where $\mathrm{x} \ldots \ldots, \mathrm{R}_{5}$.

Multi-Layer Perception (MLP) and Radial Basis function (RBF) networks are the common traditional neural networks [13]. In this paper (MLP) network is used, which is consist from three layers (input layer, hidden layer and output layer) as shown in Fig. 7.

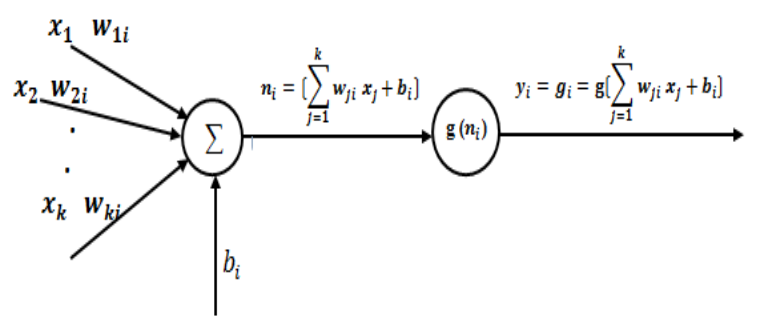

Fig. 7. The single node of the MLP

Where: $\mathrm{i}$ is the neuron number and $\mathrm{x}_{\mathrm{k}}$ is the input where $k=1, \ldots, K . \quad\left(n_{i}\right)$ is the result of multiplied the inputs by weights which is summed with each other with the bias term $b_{i}$. This result becomes the input of the activation function $(\mathrm{g})$. This function is considered to be a relay function. The sigmoid function or tangent hyberbolic ( $\tanh$ ) 
are widely used rather than the relay function [13]. The tangent function is given as:

$\tanh (x)=\frac{1-e^{-x}}{1+e^{-x}}$

In this paper our neural design is only single node of MLP networks with single input without delay, the integral derivative (ID) is consider as input to this neural controller as shown in Fig. 8, the output of neural network is multiply by proportional gain $\mathrm{K}_{\mathrm{p}}$, manual train is used to determine the value of the bias and weight.

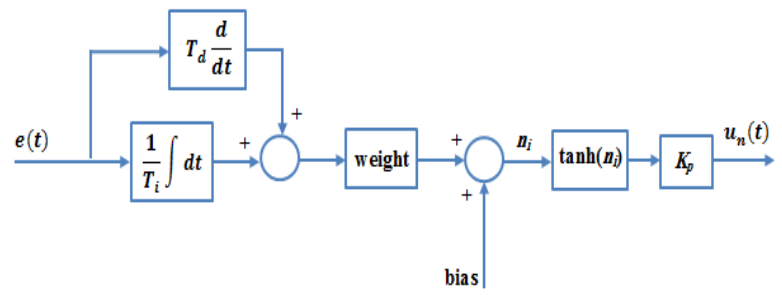

Fig. 8. Single input-single output NID controller

\section{Simulation Results and Discussion}

In this section, different simulations for the nonlinear robotic leg model with (PI+2D, PD+NID, FPD+ID, and the suggested FPD+NID) are carried out for step and multi-step input. The matlab simulink connection for the nonlinear leg model with the suggested controller is shown in Fig. 9. FPD+NID.

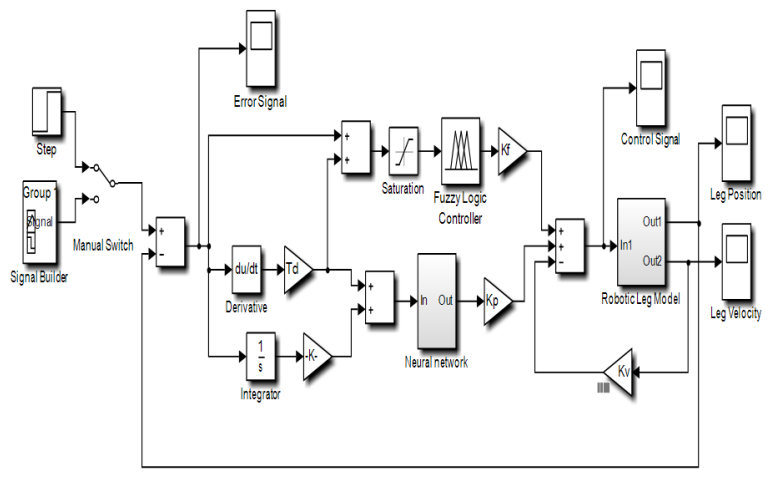

(a)

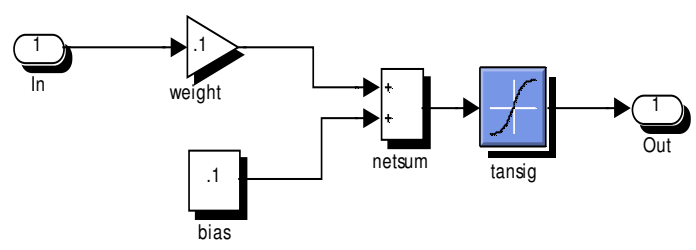

(b)

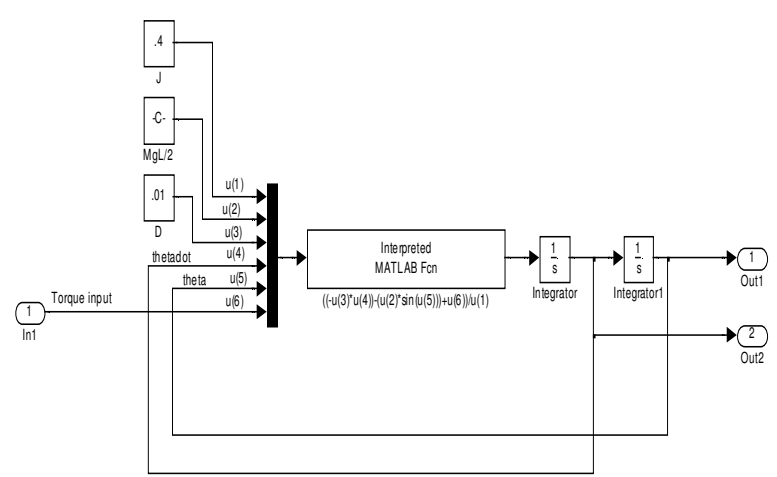

(c)

Fig. 9. Matlab simulink connection for the robotic leg with the suggested hybrid controller; (a): complete connection; (b): neural network connection; (c): nonlinear leg model connection

The controller's parameters are given by Table 2 .

Table 2,

Controller parameters.

\begin{tabular}{ccccccc}
\hline $\mathbf{K}_{\mathbf{p}}$ & $\mathbf{T}_{\mathbf{i}}$ & $\mathbf{T}_{\mathbf{d}}$ & $\mathbf{K}_{\mathbf{i}}$ & $\mathbf{K}_{\mathbf{d}}$ & $\mathbf{w}_{\mathbf{i}}$ & $\mathbf{b}_{\mathbf{i}}$ \\
\hline 14 & 2 & 0.0357 & 24 & 0.5 & 0.1 & 0.1
\end{tabular}

The different simulations are presented as follows:

i. Simulation (1): In this simulation, the controlled robotic leg is tested by step input with magnitude 2 and the results of this case (angular position, velocity, error, and control signal) which are given in Fig. 10 show that the position and velocity tracking is fairly good with nearly zero steady state error for all controller specially the our suggested FPD+NID controller.

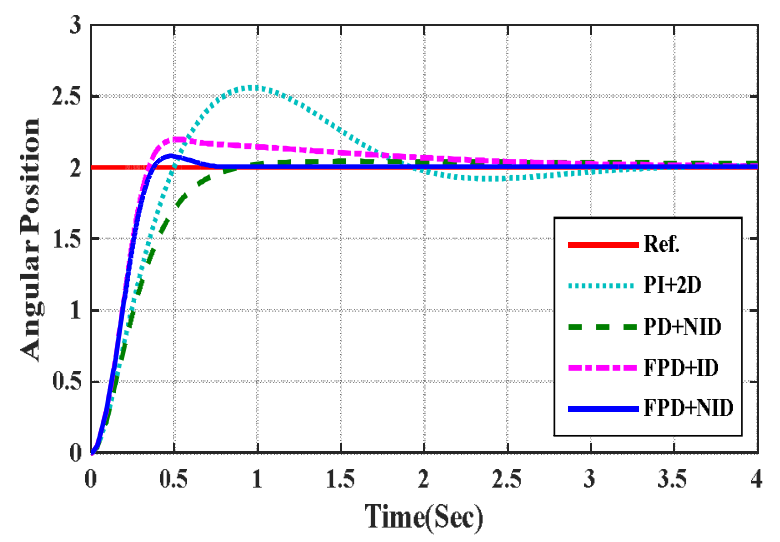

(a) 


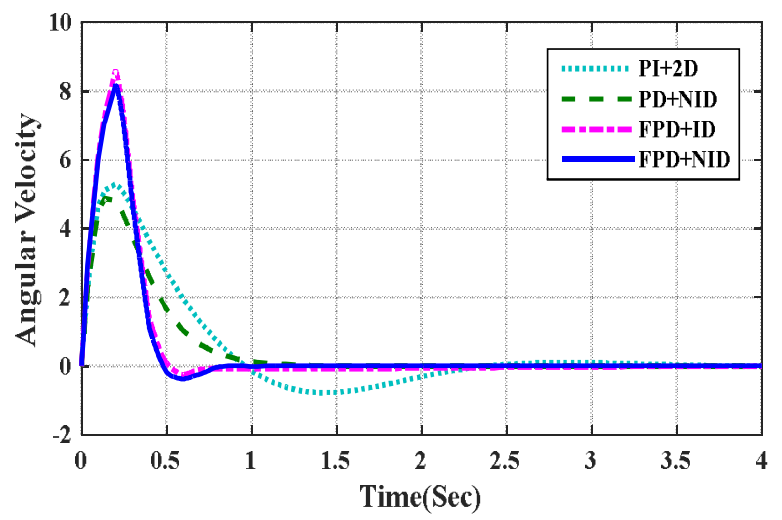

(b)

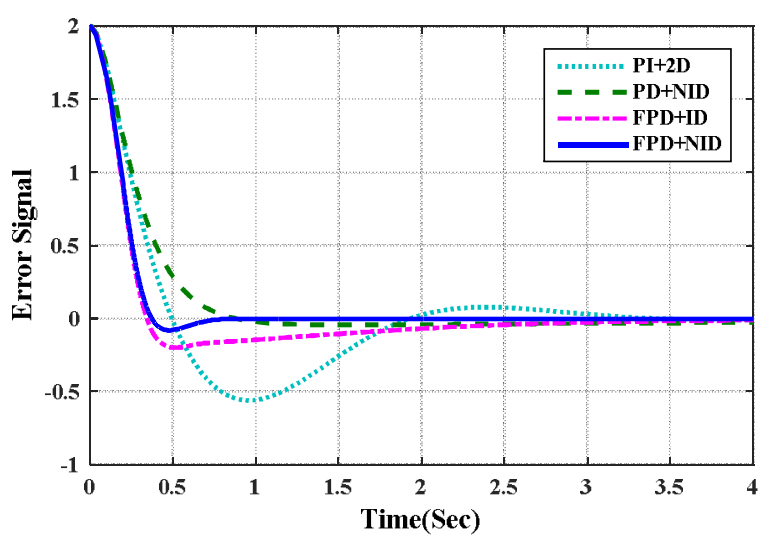

(c)

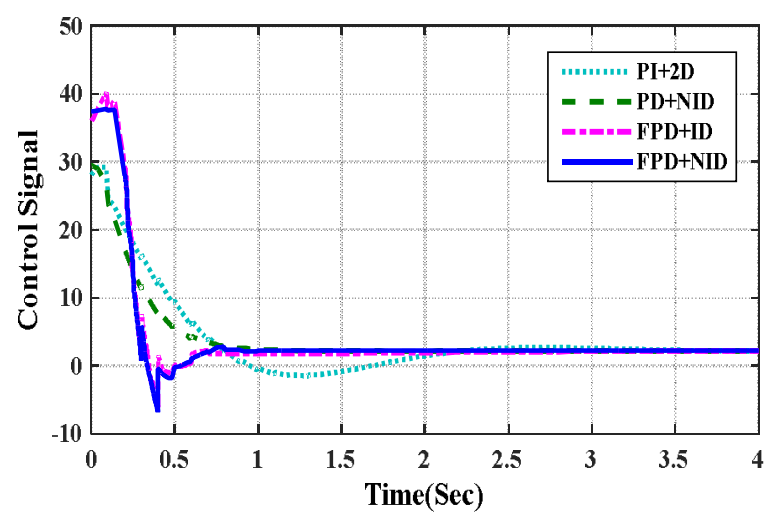

(d)

Fig. 10. Simulation results for robotic leg controlled by (PI+2D, PD+NID, FPD+ID, FPD+NID) with step input of mag.2: (a) angular position; (b) angular velocity; (c) error signal; (d) control signal

ii. Simulation (2): in this simulation, the controlled robotic leg is tested by unity step input with nonlinear sinusoidal disturbance $($ dis $=0.1 \sin (20 t))$ in addition to $10 \%$ parameter uncertainty in $\mathrm{J}, \mathrm{D}$, and $\mathrm{M}$ parameters are introduce during the simulation period. The results of this case are illustrate in Fig. 11, these results show that the robotic leg is remain stable even with disturbance and uncertainty with very small steady state error and the disturbance is remove from the response of the angular position while it is not rejected very well from the response of the velocity see Fig. 11 b.

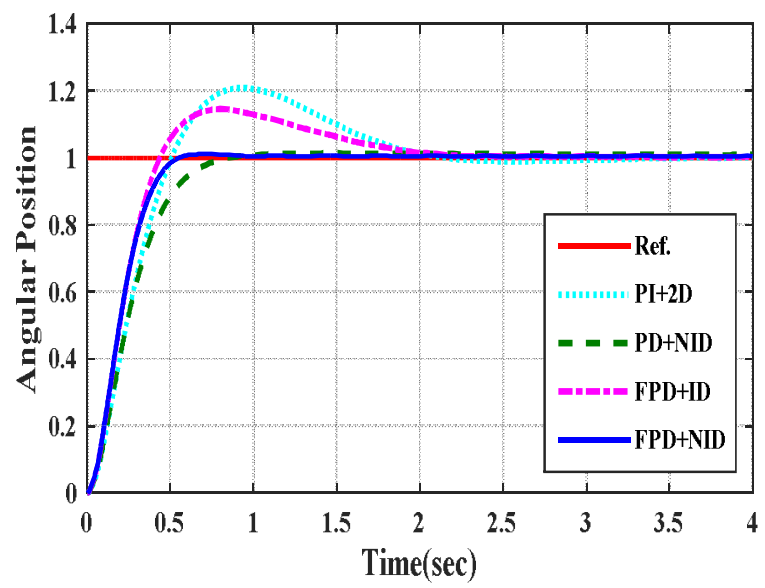

(a)

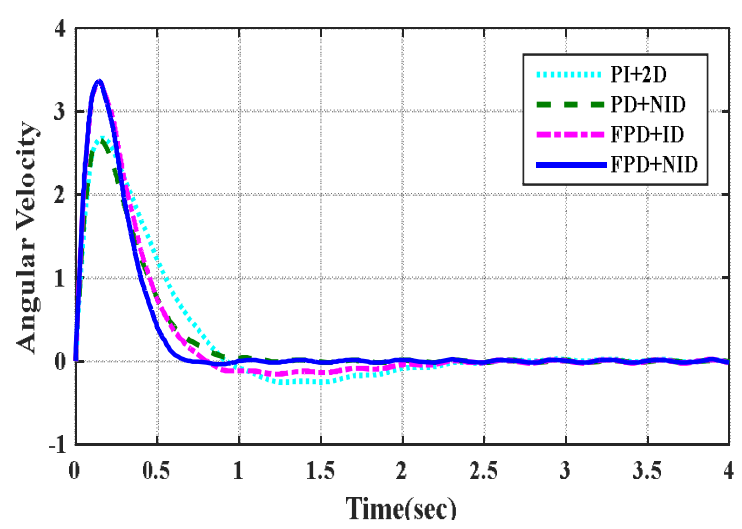

(b)

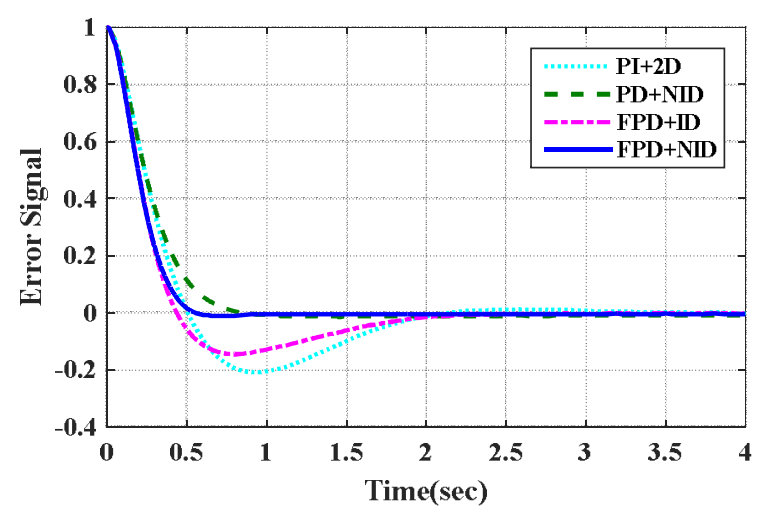

(c) 


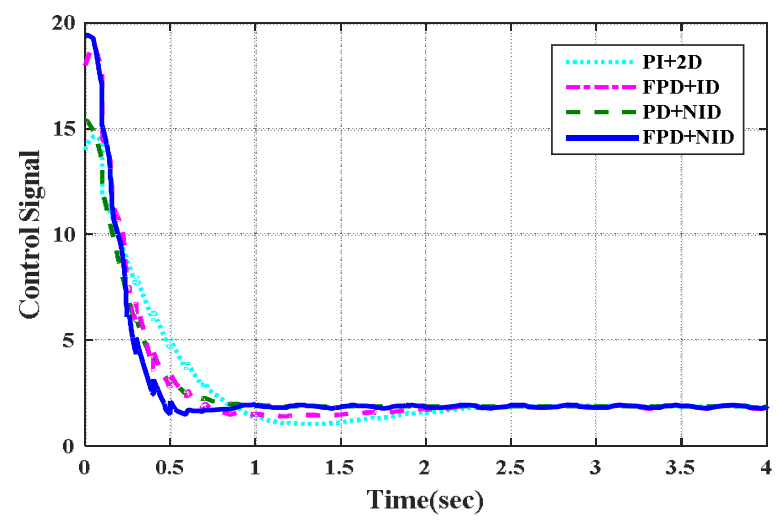

(d)

Fig. 11. Unity step simulation results with nonlinear sinusoidal disturbance and parameter uncertainty: (a) angular position; (b) angular velocity; (c) error signal; (d) control signal

iii. Simulation(3): in this simulation, the controlled robotic leg is tested by multi step input without disturbance or parameter uncertainty as shown in Fig. 12, and the results for this simulation are shown in Fig. 13, Fig.14, Fig.15, and Fig.16, these results show when sudden change occur in input signal and hence the mathematical model of robotic leg is nonlinear, the system become unstable with PI+2D, PD+NID, and FPD+ID controllers while it remain maintain the stability with efficient performance with the suggested FPD+NID controller (nearly zero steady state error with no overshoot and fast settling time).

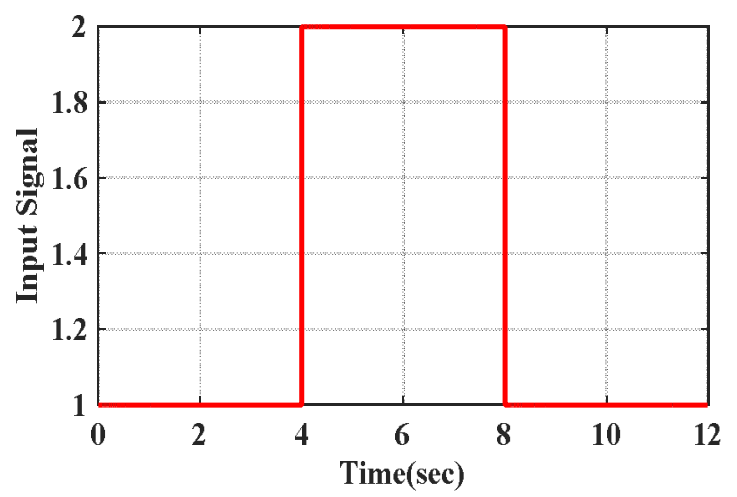

Fig. 12. Multi-step input of mag.2.

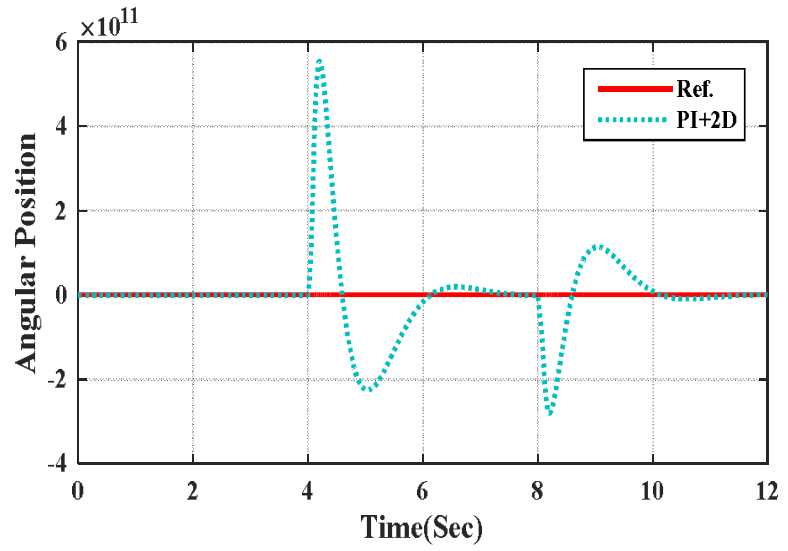

(a)

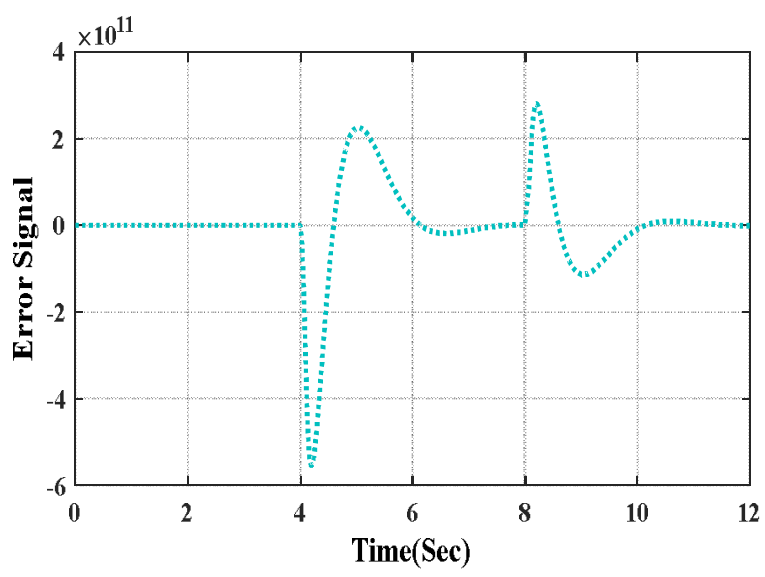

(b)

Fig. 13. Simulation results for robotic leg controlled with PI+2D and multi-step input of mag.2: (a) angular position, (b) error signal.

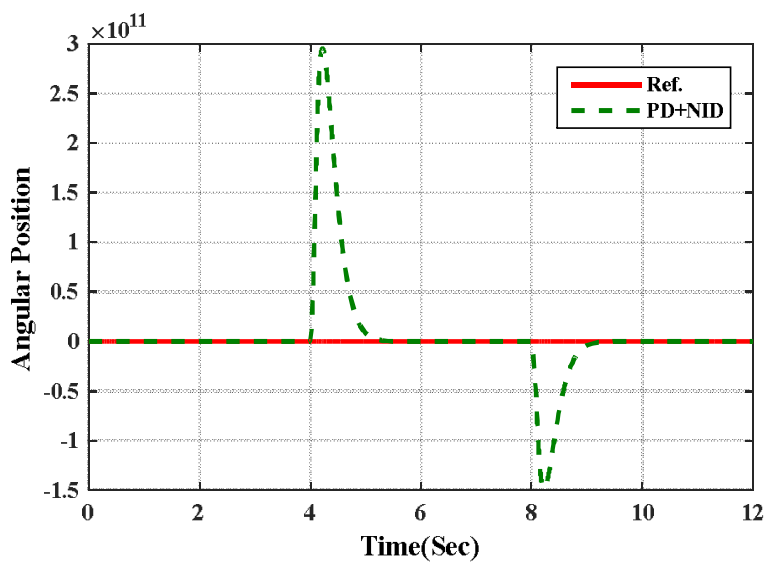

(a) 


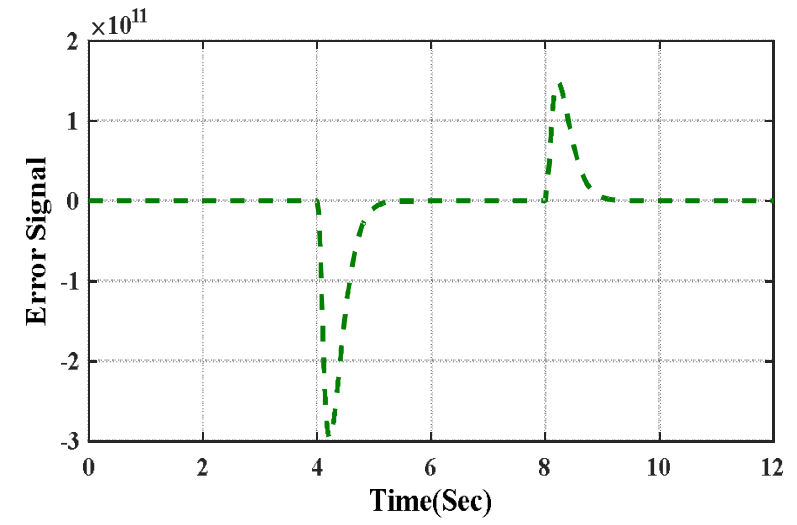

(b)

Fig. 14. Simulation results for robotic leg controlled with PD+NID and multi-step input of mag.2: (a) angular position, (b) error signal.

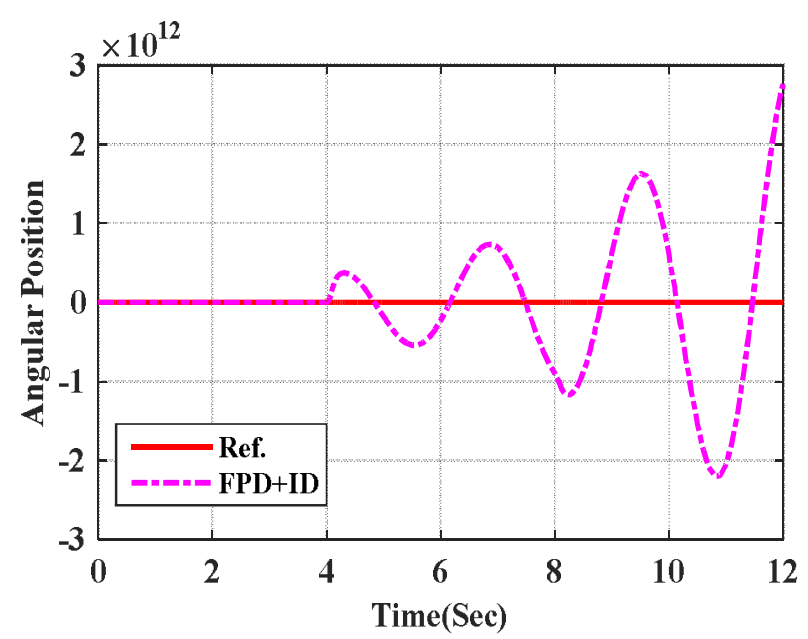

(a)

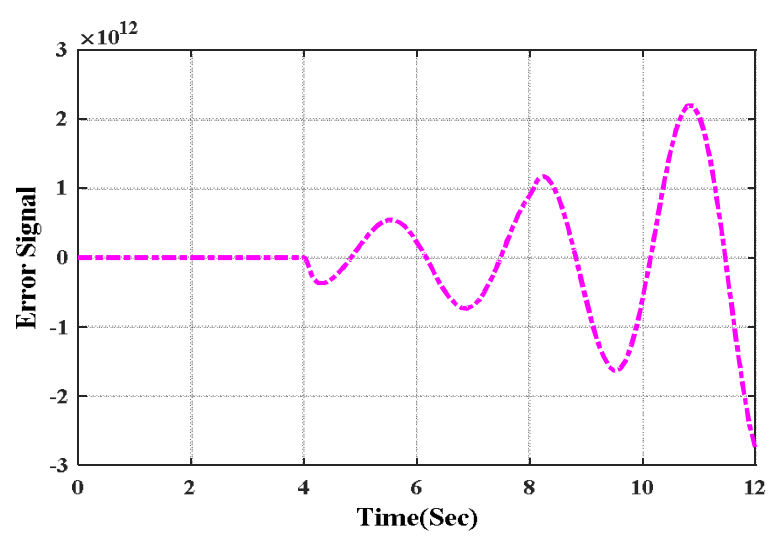

(b)

Fig. 15 Simulation results for robotic leg controlled with FPD+ID and multi-step input of mag.2: (a) angular position, (b) error signal.

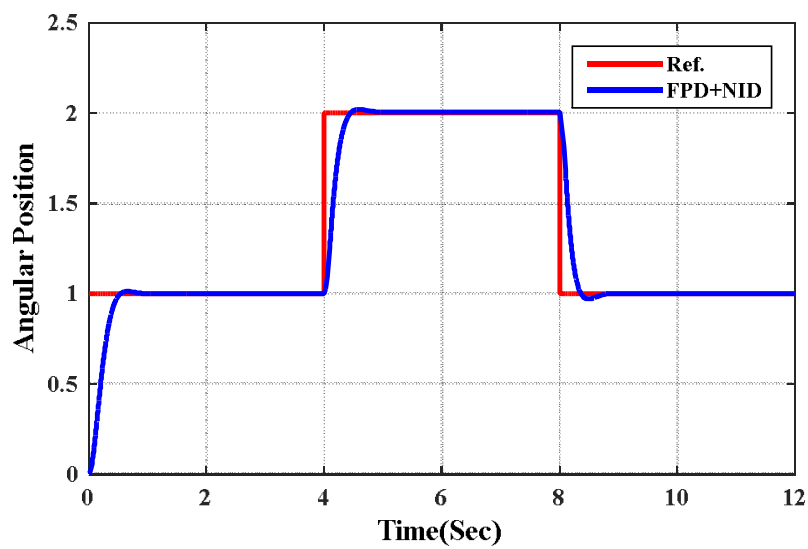

(a)

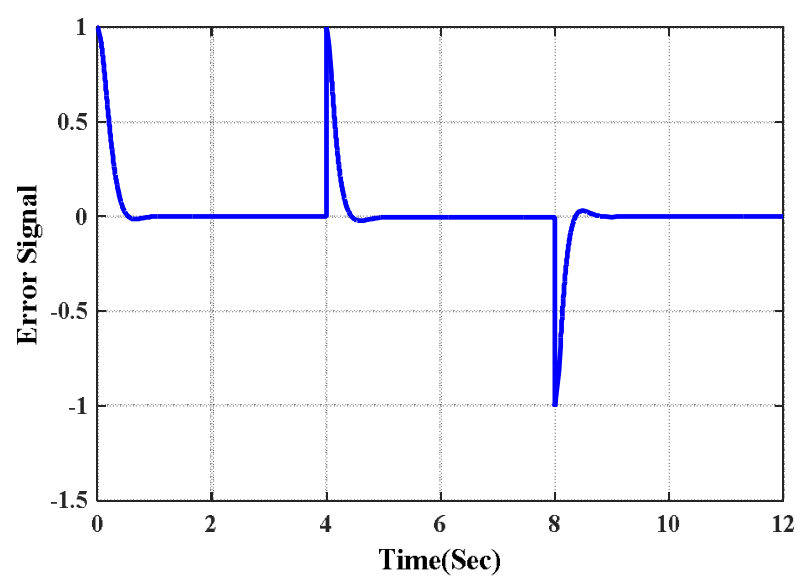

(b)

Fig. 16. Simulation results for robotic leg controlled with FPD+NID and multi-step input of mag.2: (a) angular position, (b) error signal.

\section{Conclusion}

A simple hybrid neural fuzzy controller is designed to improve the performance of human robotic leg model which is SISO. This controller consists from fuzzy proportion derivative (FPD) controller and single node neural integral derivative (NID) controller. The different simulation results with / without parameter uncertainty and nonlinear disturbance show that the suggested FPD+NID controller is better than $\mathrm{PI}+2 \mathrm{D}, \mathrm{PD}+\mathrm{NID}$ and FPD+ID controllers. 


\section{References}

[1] Kenji Kaneko, Shuuji Kajita, Fumio Kanehiro, Kazuhito Yokoi, Kiyoshi Fujiwara, Hirohisa Hirukawa, Toshikazu Kawasaki, Masaru HIRATA, and Takakatsu Isozumi, Design of Advanced Leg Module for Humanoid Robotics, Project of METI, International Conference on Robotics Automation, Washington, DC, May, 2002.

[2] Alexander Schepelmann, Michael D. Taylor, Hartmut Geyer, Development of a Testbed for Robotic Neuromuscular Controllers, Robotics: Science and System VIII, Jan 2012.

[3] A. Abraham et al., Neuro-Fuzzy Methods for Modeling and Identification, Recent advances in intelligent paradigm and applications, springer-verlag, Berlin Heidelbe, 2003.

[4] Wei-Yen Wanga, Yih-Guang Leub, Tsu-Tian Lee, Output-feedback control of nonlinear systems using direct adaptive fuzzy-neural controller, Fuzzy Sets and Systems 140, 341358, 2003.

[5]Dr. Zs. J. Viharos, K. B. Kis, Survey on Neuro-Fuzzy Systems and their Applications in Technical Diagnostics, 13th IMEKOTC10 Workshop on Technical Diagnostics Advanced measurement tools in technical diagnostics for systems' reliability and safety, Warsaw, Poland, June 26-27, 2014.

[6] A. Aldair and W. J. Wang, Adaptive Neuro Fuzzy Inference Controller for Full Vehicle Nonlinear Active Suspension Systems, School of Engineering and Design, Iraq $J$. Electrical and Electronic Engineering, Vol.6 No.2, 2010.

[7] A. Fahmy , A.M. Abdel Ghany, Neuro-fuzzy inverse model control structure of robotic manipulators utilized for physiotherapy applications, Ain Shams Engineering Journal, 805-829, 2013.

[8] Chun HtooAung, KhinThandarLwin, and Yin Mon Myint, Modeling Motion Control System for Motorized Robot Arm using MATLAB, World Academy of Science, Engineering and Technology 42, 2008.

[9] Abdur Raquib Ridwan, Md. Ibnea Sina Bony and Ishtiza Ibne Azad, Performance Analysis of MPC in the Control of a Simple Motorized Nonlinear Model of a Robotic Leg, International Journal of Computer Applications (0975 - 8887), Volume 54, No. 11, September 2012.
[10] Richard L. Shell Ernest L. Hall, Handbook of Industrial Automation, University of Cincinnati Cincinnati, Ohio, New Yourk, 2000.

[11] Nidhi K., Simulation Studies on Hybrid Fuzzy-PI Controllers for DC Motor Control, Master thesis of Engineering in Control \& Instrumentation, Maharishi Dayanand University, Rohtak Haryana, 2006.

[12] C. Yen, F. Wen, and M. Ouyang, Nonlinear Positioning Compensator of a Novel Thin-Disc Ultrasonic Motor using Fuzzy Sliding Mode Control, International Journal of Applied Science and Engineering, Vol. 2, No. 3, pp. 257-276, 2004.

[13] [Heikki N. Koivo, Neural Networks: Basics using MATLAB, Neural Network Toolbox, February 1, 2008. 


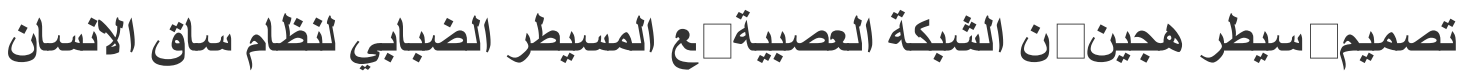

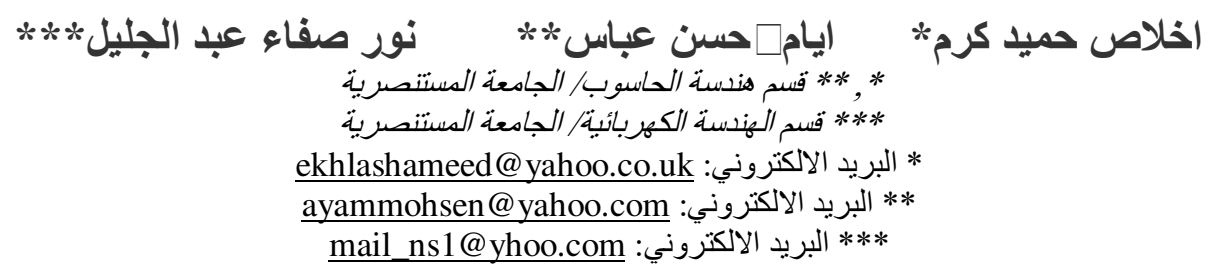

في هذا البحث, الساق الروبوتية البشرية التي يمكن ان تمثل رياضيا بوساطة النموذج التفاضلي غير الخطي احادي الادخال-احادي الاخر اج (SISO)

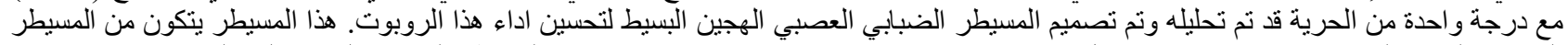

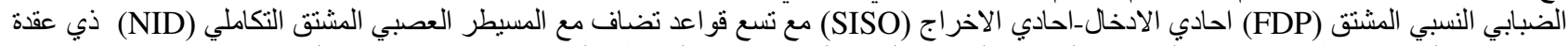

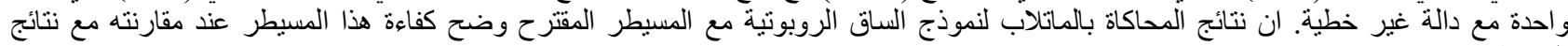
المسيطر ات دال 